\title{
Mitigation of Low Frequency Oscillations by Optimal Allocation of Power System Stabilizers: Case Study on MEPE Test System
}

\author{
Wai Myat Thu, Kyaw Myo Lin \\ Department of Electrical Power Engineering, Mandalay Technological University, Patheingyi, Mandalay, Myanmar \\ Email: waimyatthuep@mtu.edu.mm,kmlinep@mtu.edu.mm
}

How to cite this paper: Thu, W.M. and Lin, K.M. (2018) Mitigation of Low Frequency Oscillations by Optimal Allocation of Power System Stabilizers: Case Study on MEPE Test System. Energy and Power Engineering, 10, 333-350.

https://doi.org/10.4236/epe.2018.108021

Received: July 17, 2018

Accepted: August 12, 2018

Published: August 15, 2018

Copyright ( 92018 by authors and Scientific Research Publishing Inc. This work is licensed under the Creative Commons Attribution International License (CC BY 4.0). http://creativecommons.org/licenses/by/4.0/

\begin{abstract}
The increases in power network and weak tie-line have led power system oscillation problems. To improve the oscillatory stability, installing the power system stabilizer (PSS) with optimal allocation is considered due to excessive cost. This paper recommends the suitable PSS locations by using eigenvalue analysis and participation factor to enhance the system oscillation damping. The effects of installed PSSs in damping local and inter-area modes of oscillations are confirmed through time domain simulation results. The effectiveness of proposed approach is tested and validated on MEPE test system. Robustness of stabilizers against dynamic response of generator speed deviation, rotor angle deviation, and response of mechanical power are observed to access the performances of PSSs.
\end{abstract}

\section{Keywords}

Eigenvalue Analysis, MEPE Test System, Oscillation Mode, Optimal Location of PSS, Participation Factor

\section{Introduction}

Modern power system can be characterized by widespread system interconnections composed of multiple machines connected by the transmission. In the interconnected large electric power networks, there have been always unwanted unprompted system oscillations at very low frequencies in order of $0.2-2.0 \mathrm{~Hz}$ [1]. These low frequency oscillations are major constraints for power transfer increase between regions in a power system, mainly between weakly interconnected areas during heavy load conditions. It is required to damp out the unwanted power swing by changing output power, controlling the excitation value 
and reducing the power oscillations in order to be system stable.

The development of Power System Stabilizer (PSS) brought in power oscillation problems [2]. In view of the potentially high cost of using many PSS and to assess its effectiveness in damping poorly damped swing modes, the identification of the optimum site of PSS is still becoming an important task. The concept of the participation factor [3] was used to choose the machine having the greatest participation factor with the most poorly damped swing mode for the optimum allocation of PSS.

The aim of this paper is to propose the allocation of PSS in MEPE test system for optimal damping and power system stability enhancement. In this paper, participation factor analysis is applied to determine locations of PSS. On the other hand, eigenvalue analysis describes the oscillatory problem associated with stable or unstable limit cycles in case study network. The rest of the paper is organized as follows: characteristics of low frequency oscillation problem are analyzed in Section 2. Section 3 presents the analysis techniques and simulation tool with subsection. Then, a description of MEPE test system for case study is given in Section 4. In Section 5, the time domain simulation results are discussed in detail. Finally, the conclusions are drawn based on the simulated results.

\section{Analysis of Low Frequency Oscillation}

Electric power sources are located in remote places where power is transferred over long transmission lines causing wide area power interchanges. Under such conditions, there is a concern that the stability of the system will be affected not only by local power swing oscillations (local modes), but also by long-cycle oscillation phenomena (inter-area modes) which will influence the whole system [3]. Moreover, the weak tie-lines and the nature of the longitudinal structure are some roots for low frequency oscillations which having a frequency between 0.1 $2.0 \mathrm{~Hz}$.

Local modes are associated with the swinging of generator units at a generating station with respect to the rest of the power system. Oscillations occurred only to the small part of the power system. Typically, the frequency range is $1-2$ $\mathrm{Hz}$. Local oscillations often occur when a fast exciter is used on the generator. Inter-area modes are associated with swinging of many machines in one part of the system against machines in other parts. It generally occurs in weak interconnected power system through long tie-lines. Typically, frequency range is 0.1 $1 \mathrm{~Hz}$. Inter-area oscillations will appear as the systems loading is increased across the weak transmission links in the system which characterizes these oscillations [4]. If not controlled, these oscillations will lead to total or partial power interruption.

\section{Analysis Techniques and Simulation Tools}

\subsection{Eigenvalue Analysis of Multi-Machine Power System}

Linear analysis is widely used to analyze oscillatory behavior of power systems. 
The system is linearized about an operating point and typically, it involves computation of eigenvalues, eigenvectors and system modes from state-space representation of power system model [3]. This is also termed as "Small Signal Stability Analysis", "Modal Analysis" or "Eigenvalue Analysis" that are used in this paper for studying oscillatory modes. In the process of evaluating the eigenvalues, it is important to model loads, controllers and other power system components: statically and dynamically.

A general mathematical model of power system is given by the following equation:

$$
\begin{aligned}
\dot{x} & =f(x, y, l, p) \\
0 & =g(x, y, l, p)
\end{aligned}
$$

where, $x$ is a vector of state variable; $y$ is a vector of algebraic variables; 1 and $p$ are uncontrollable and controllable parameters, respectively. Machine and control dynamics will be included in the differential equations while basic load flow and other network equations will be included in algebraic equations. Equilibrium point or operating point of the system can be found by simultaneously solving differential and algebraic equations (DAEs) given in (1).

Once the equilibrium point is known, DAE model can be linearized around the equilibrium point as given in (2).

$$
\left[\begin{array}{c}
\Delta \dot{x} \\
0 \\
0
\end{array}\right]=\left[\begin{array}{ccc}
A^{\prime} & B_{1}^{\prime} & B_{2}^{\prime} \\
C_{1}^{\prime} & D_{11}^{\prime} & D_{12}^{\prime} \\
C_{2}^{\prime} & D_{21}^{\prime} & D_{22}^{\prime}
\end{array}\right]\left[\begin{array}{c}
\Delta x \\
\Delta Y_{C} \\
\Delta Y_{B}
\end{array}\right]+\left[\begin{array}{c}
E_{1} \\
0 \\
0
\end{array}\right] \Delta U
$$

where, $D_{22}^{\prime}$ is the load flow Jacobian $\left(J_{L F}\right)$ modified by the load representation and $\left[\begin{array}{ll}D_{11}^{\prime} & D_{12}^{\prime} \\ D_{21}^{\prime} & D_{22}^{\prime}\end{array}\right]=J_{A E}^{\prime}$ is the network algebraic Jacobian.

The system matrix, $A_{s y s}$ obtained from (2) is

$$
\Delta \dot{x}=A_{s y s} \Delta X+E \Delta U
$$

where, $\left[A_{s y s}\right]_{7 \mathrm{~m} \times 7 \mathrm{~m}}=\left[A^{\prime}\right]-\left[\begin{array}{ll}B_{1}^{\prime} & B_{2}^{\prime}\end{array}\right]\left[J_{A E}^{\prime}\right]^{-1}\left[\begin{array}{c}C_{1}^{\prime} \\ C_{2}^{\prime}\end{array}\right]$

Matrix $A_{s y s}$ in (3) is referred to as reduced system state matrix. Small signal stability or steady state stability of the equilibrium point of the system can be analyzed by looking at the eigenvalues of matrix $A_{s y s}$ Eigenvalues are given by the following equation and the number of eigenvalues depends on the dimension of matrix, A or the number of state variables considered in the system [2].

$$
\operatorname{det}(A-\lambda I)=0
$$

where, $\lambda$ is eigenvalue. In order to the system be stable or oscillation free, all the eigenvalues should be located in the open left half plane. If at least one of the eigenvalues has positive real part, the system is said to be unstable. More specifically, in oscillatory unstable cases, a pair of complex eigenvalues $(\lambda=\sigma \pm j \omega)$ will appear with positive real part [2]. 


\subsection{Damping Low Frequency Oscillation}

The damping coefficient and oscillation frequency can be used to evaluate the damping effects of the power system stabilizers on the power oscillation [4]. The damping coefficient $\left(\zeta_{i}\right)$ and oscillation frequency $\left(f_{i}\right)$ of the $i^{\text {th }}$ eigenvalue are defined with the following set of equation:

$$
\xi_{i}=\frac{-\sigma_{i}}{\sqrt{\sigma_{i}^{2}+\omega_{i}^{2}}}, f=\frac{\omega}{2 \pi}
$$

It is obvious that the higher damping ratio and the lower oscillation frequency, the better damping effects to enhance the stability of the power system [5]. The aim of oscillation damping is to achieve 5\% damping for all modes overall operating conditions under consideration.

\subsection{Determining the Dominant Generator by Utilizing Participation Factor}

In order to study small signal stability, it is necessary to know which state variables significantly participate in the selected modes. Participation factorisa measure of relative participation of $k^{\text {th }}$ states in the $I^{\text {th }}$ modes [6], and the participation factor analysis aids in the identification of how each state variable affects a given mode. The participation factor can be defined as,

$$
P_{i}=\left[\begin{array}{llll}
P_{1 i} & P_{2 i} & \ldots & P_{n i}
\end{array}\right]^{\prime}=\left[\begin{array}{lllll}
\Phi_{1 i} \Psi_{i 1} & \Phi_{2 i} \Psi_{i 2} & \ldots & \Phi_{n i} \Psi_{i n}
\end{array}\right]^{\prime}
$$

From (6), participation factor $p_{k i}$ of any $k^{\text {th }}$ state variable in any $i^{\text {th }}$ mode can be measured as,

$$
P_{k i}=\Phi_{k i} \Psi_{i k}
$$

where, $\Phi_{k i}$ is the $k^{\text {th }}$ entry of the right eigenvetor of $I^{\text {th }}$ mode and $\psi_{i k}$ is the $k^{\text {th }}$ entry of the left eigenvetor of $i^{\text {th }}$ mode.

The machine containing positive real part of the participation factor of its speed and rotor angle states may be a potential candidate for placement of PSS [6].

\subsection{Simulation Tool}

In this paper, state variables initialization, modal analysis (eigenvalues analysis) and time domain simulations were carried out using the Power System Analysis Toolbox (PSAT) [7]. PSAT has been used for all the linear and transient analysis. It is an open source MATLAB and GNU/Octave-based software package for analysis and design of small to medium size power systems [8]. It can perform Power Flow (PF), Continuation Power Flow (CPF), Optimal Power Flow (OPF), Time Domain Simulation, and Small Signal Stability Analysis.

In this tool, machine and associated controls: such as governor, exciter and PSS are modeled according to pre-specified data format and are also stored in the data file [7]. Linearization algorithm has been implemented in a built-in routine which performs linearization of the network based on a load flow analysis. 
Eigenvalues, left and right eigenvectors, system modes specified by damping coefficient and frequency, participation factors and other required information for linear analysis are available upon running small signal stability routine through dynamic analysis. PSAT has several tools with graphical features, of which the transient stability and small signal stability tools were used to get the simulated results presented in this paper.

\section{System Description and Modeling of System Components}

The MEPE test system is currently modeled as 202 buses fed by 40 generating stations. The Myanmar electricity network has the characteristics of bearing having generation by hydro power in the northern region while supplying large consumption in the southern region through the tie-lines [9].

\subsection{Modeling of Power System Components}

This section presents the models used for the generator, turbine and speed governor, automatic voltage regulators and power system stabilizers followed by a detailed description of the MEPE test system. All models used are documented in the PSAT manual [8].

\subsubsection{Generator Models}

Two synchronous machine models are used for the test system: three-rotor windings for the salient pole machines of hydro power plants and four-rotor windings for the round-rotor machines of thermal plants. MEPE test system is composed of fifteen thermal generating stations and twenty-five hydro generating stations. The thermal generators are denoted by $G 6, G 7$ and $G 13$ to $G 18$, whereas hydro generators are denoted by $G 1$ to $G 5, G 8$ to $G 12, G 19$ and $G 20$. These two types of generators are described by five and six state variables, respectively: $\delta, \omega, e_{q}^{\prime}, e_{q}^{\prime \prime}, e_{d}^{\prime \prime}$, and with an additional state $e_{d}^{\prime}$ for the six-state variables [8].

\subsubsection{Automatic Voltage Regulator}

Three simple different types of AVRs are defined in PSAT and AVR Type II is the standard IEEE model 1. The same model of AVR (Type II), as shown in Figure 1 , is used for all generators but with different parameters.

\subsubsection{Turbine and Governor Models}

In PSAT, there are six models of turbine and governors (TG), in which Model 1 and Model 3 are used in this study. For conventional thermal generating units, Model 1 turbine governors (TG) were employed while the hydroelectric units use Model 3 TG [10]. Model 3 consists of a typical hydro turbine governor modeland a linearized hydro turbine model. The main difference between turbine models for thermal and the hydroturbine and governor models is the introduction of the gate position. Block diagrams of turbine and governor models for Model 1 and Model 3 are depicted in Figure 2 and Figure 3, respectively. 


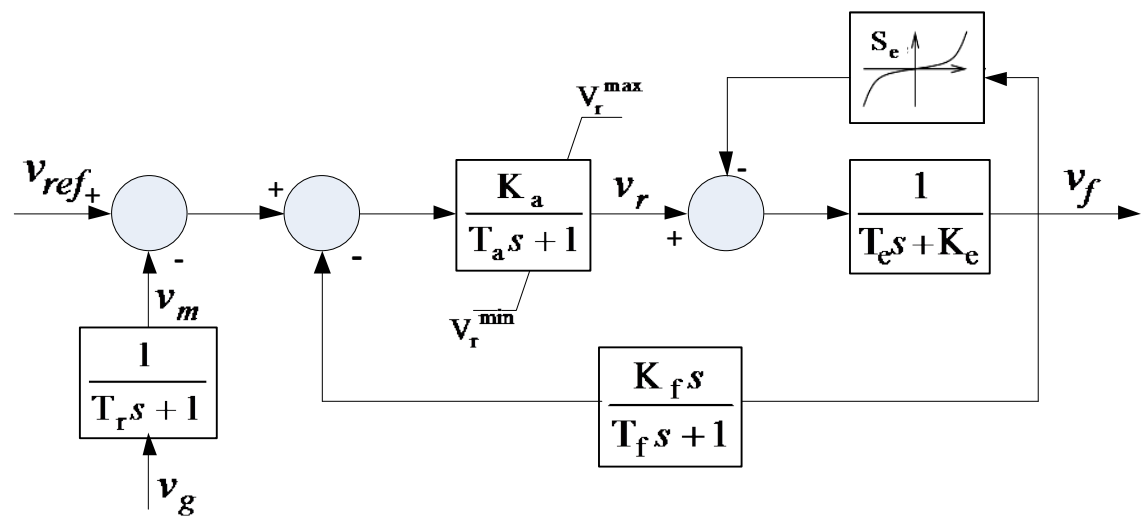

Figure 1. Exciter model.

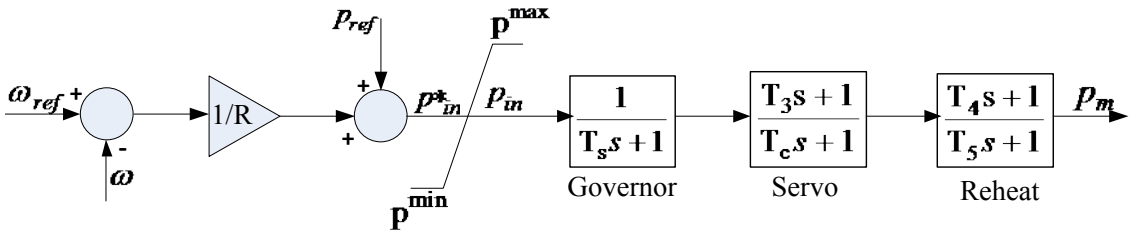

Figure 2. Turbine governor model used for thermal generators: Model 1.

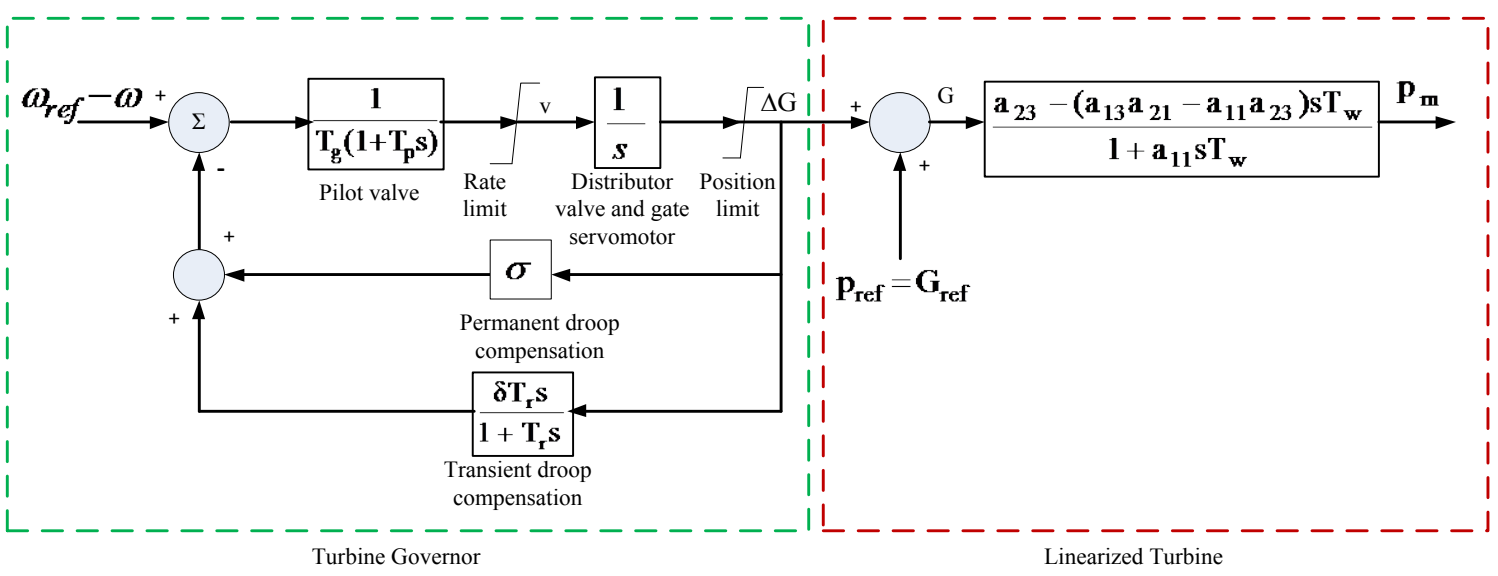

Figure 3. Turbine governor model used for hydro generators: Model 3.

\subsection{Case Study Network}

K. M. Lin, et al. presented the open source software based MEPE test system [9] and in this study, this test system is modified with recent generation and network expansion. Recently, a mixture of coal, diesel and geothermal power plants comprises the main generation profile of the grid peaking to $3040 \mathrm{MW}$ [11]. Recent developments in the southern grid of the Myanmar added a $230 \mathrm{kV}$ network in its existing $132 \mathrm{kV}$ and $66 \mathrm{kV}$ lines which originally has a total of 1300 $\mathrm{km}$. All in all, the new test system model is composed of 202 buses with a total number of lines is 232 with 76 units of transformers and 73 load points. There are total of 20 shunt capacitors and reactors are injected in MEPE network which are shown in Figure 4. The bus no. 119 (Yeywa hydro station) was chosen 


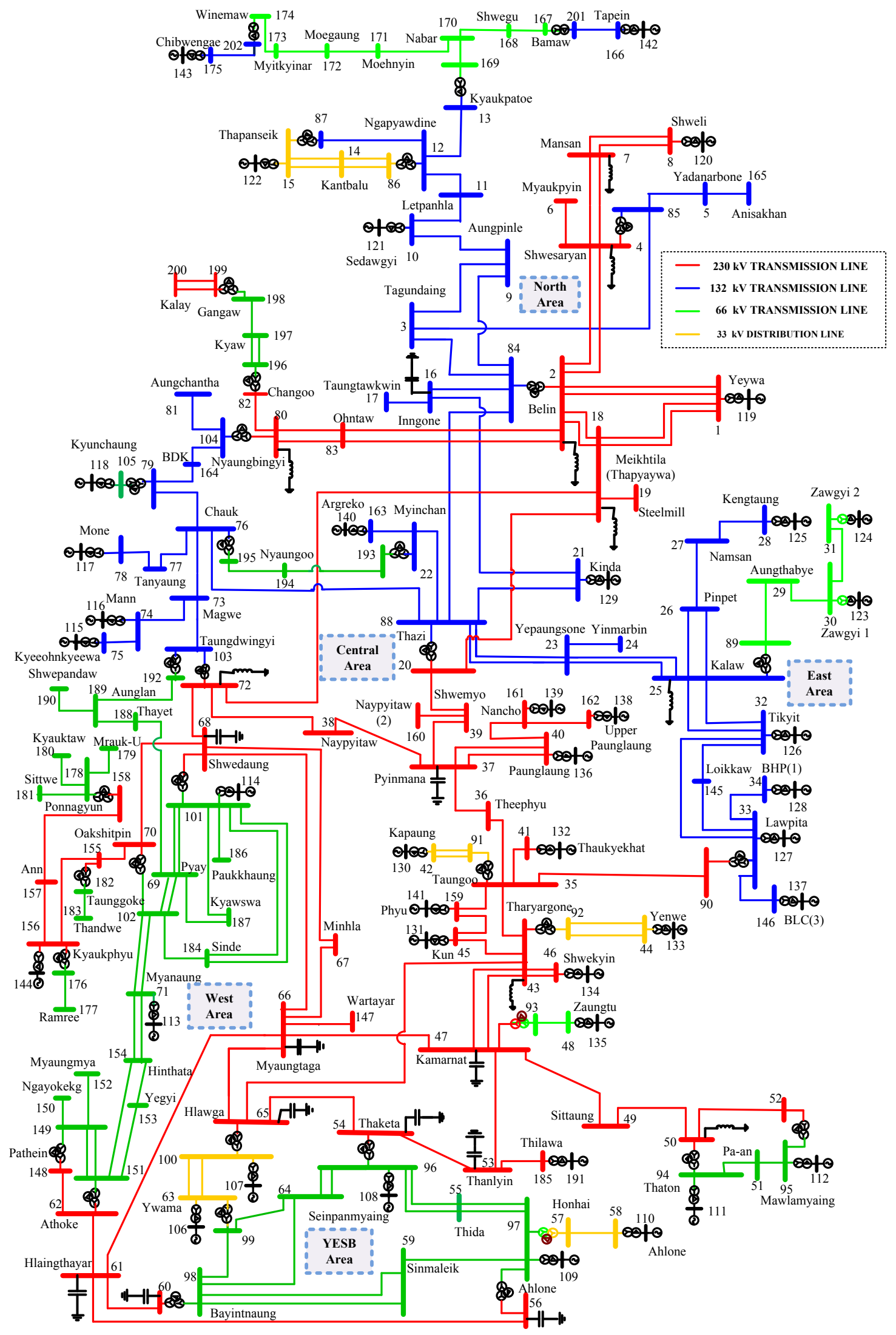

Figure 4. Single line diagram of MEPE test system. 
as the slack bus while the others are considered as voltage controlled bus.

MEPE test system is based on a system data including dynamic model in the above sections proposed by KEPCO [11] (consultant of MEPE) and the staffs of power system department of MEPE. The grid study is valid for the transmission system only and do not include the distribution network. The grid has no direct connections to other grids of neigh boring countries.

\section{Allocation of Power System Stabilizer}

\subsection{Eigenvalue Analysis of MEPE Test System}

Once the power flow problem has been solved, the eigenvalues and participation factors of the state matrix are computed in order to identify the critical modes of the system. The frequency of oscillation is derived from the imaginary part of eigenvalues while the damping ratio is derived from the real part. Damping ratios indicate "how" stable a system is; the higher the (positive) value of a damping ratio, the more stable the system is for a given oscillation.

Through dynamic analysis pane of PSAT, the plot of eigenvalues of the MEPE test system is illustrated in Figure 5.

A total of 530 states including 152 complex pairs are initialized and it can be observed in Figure 5. According to the numerical results, twenty positive eigenvalues were detected in the MEPE test system. Therefore, the MEPE test system is operating at an unstable operating condition because of positive eigenvalues. The system exhibits negative damped low frequency oscillations. There are 32 complex pairs out of 152 complexes at low frequency (frequency less than $2 \mathrm{~Hz}$ ), which are observed as critical modes. The oscillation modes with respect to oscillation frequency have been achieved based on (5) and the most influence modes are ranked in Table 1.

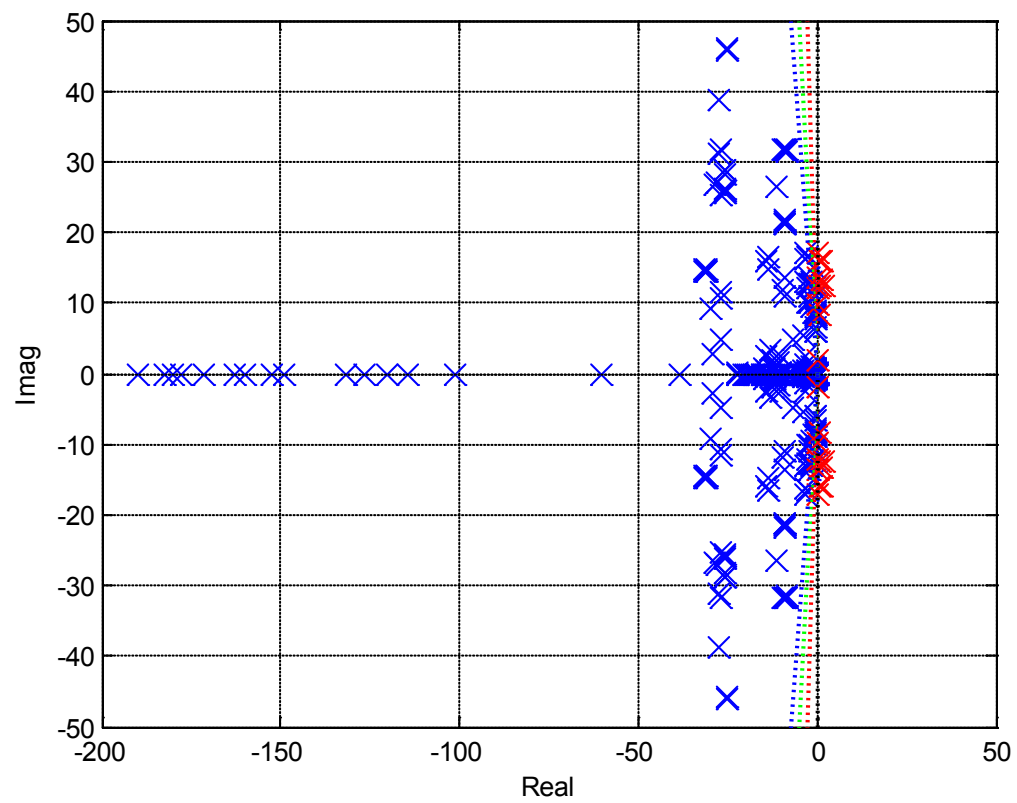

Figure 5. Eigenvalues of MEPE test system. 
Table 1. Dominant generators and states for different modes in MEPE test system.

\begin{tabular}{cccc}
\hline Mode & Eigenvalue & Frequency & Damping Ratio \\
\hline 1 & $1.5988 \pm \mathrm{j} 12.5657$ & 1.9999 & -0.1262 \\
2 & $0.75405 \pm \mathrm{j} 12.5647$ & 1.9997 & -0.0599 \\
5 & $-0.0097 \pm \mathrm{j} 12.2154$ & 1.9441 & 0.0008 \\
8 & $-0.6023 \pm \mathrm{j} 10.9343$ & 1.7403 & 0.055 \\
12 & $0.31868 \pm \mathrm{j} 9.7201$ & 1.547 & -0.0328 \\
13 & $-0.2603 \pm \mathrm{j} 9.06469$ & 1.4427 & 0.0287 \\
16 & $0.84065 \pm \mathrm{j} 8.38064$ & 1.3338 & -0.0998 \\
31 & $0.46106 \pm \mathrm{j} 1.8729$ & 0.29808 & -0.239 \\
\hline
\end{tabular}

The damping ratios in column 4 are calculated based on eigenvalues of column 2. From the above table, it can be depicted that two of these low frequency modes present damping ratio lower than $3 \%$ and some modes has negative value in damping. It means that the system is badly damp system. To improve the damping, it is needed to install PSS and its allocation is very important. Therefore, the approach for optimal PSS allocation will be mentioned in the following section.

\subsection{Determining the Dominant Generator by Utilizing Participation Factor}

As PSSs are very expensive, it is not wise to install PSS in all the generators. The rotor angle and angular speed of a generator, which have the highest participation rates on the main dominant modes, affects the power system oscillatory stability. It is required to install the PSS into these dominant generators. To determine which generator is subjected in dominant state, participation factor analysis is applied in this research. Participation factors are evaluated based on eigenvalues of MEPE test system and the obtained results concerning with participation factors are displayed in Figure 6.

As discussed in Section 5.1, the system has 530 eigenvalues. Participation factor is the relative participation of the state in the modes. To view which state variable is dominantly associated which mode, the participation factors versus state variables of all generators on the dominant oscillation modes are depicted in Figure 7.

Mode is identified by eigenvalues with respect to the range of frequency. As seen in above figure, G9, G6, G5 and G4 have the highest participation factor on Mode 19, 16, 2 and 12 among other generators. Therefore, the results of evaluating the participation factor show that G9, G6, G5 and G4 dominate the low-frequency-oscillation in the system. Moreover, G11, G32, G35 and G37 are also chosen as the candidate generators because they are also influence on oscillatory stability. Their damping ratios are very low and these participation rates are located in second highest level. The participation rates of state variables and 


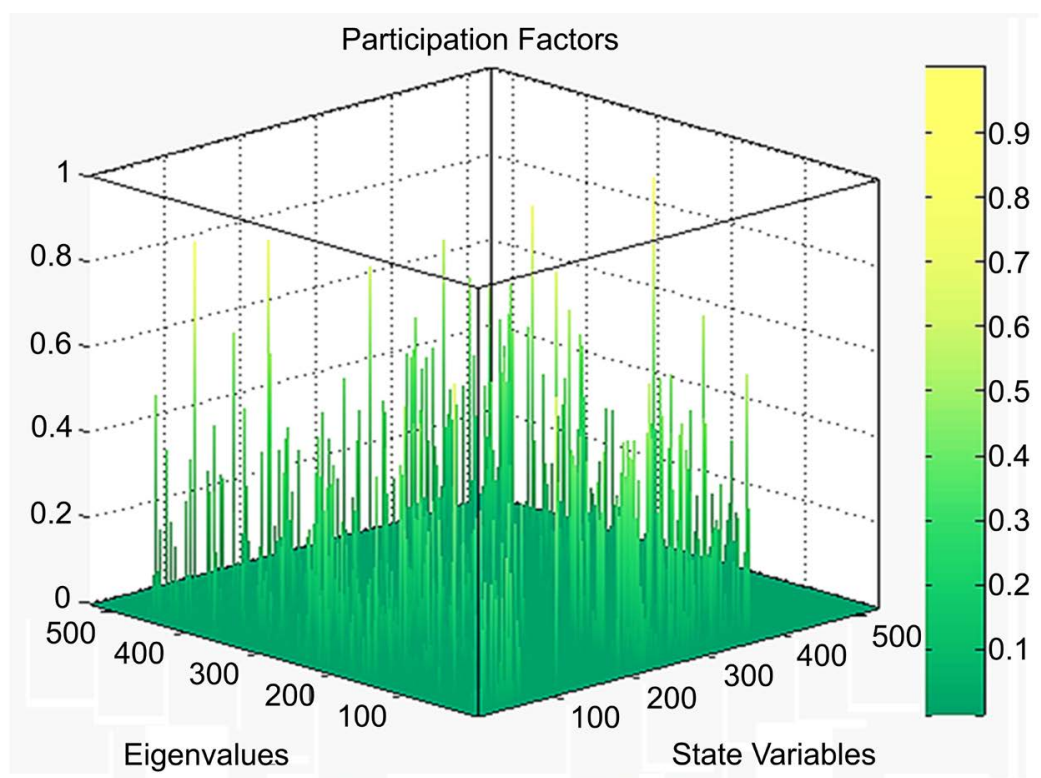

Figure 6. System participation factors versus state variables of MEPE test system.

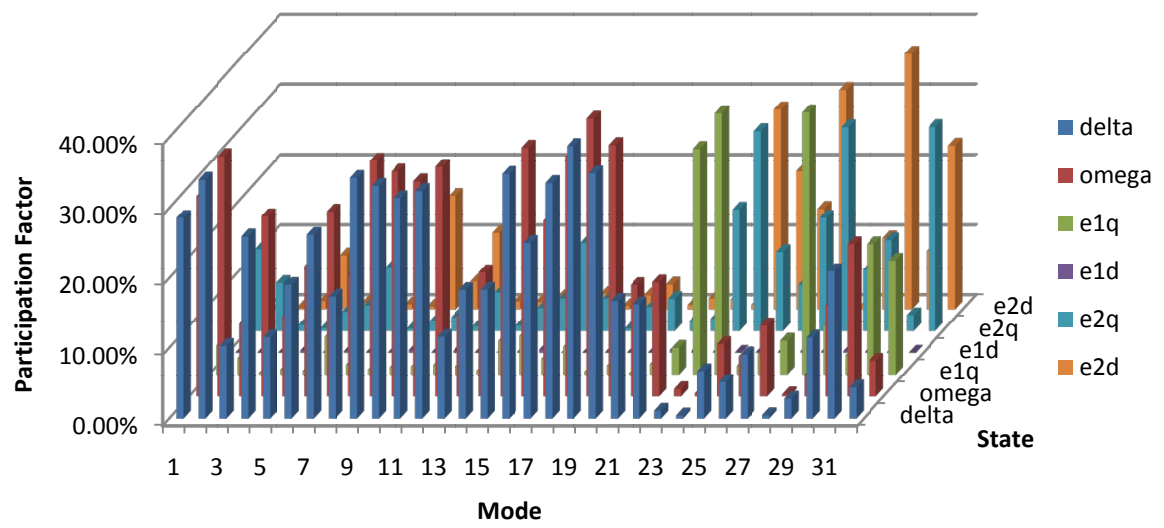

Figure 7. System participation rates of MEPE test system.

different oscillation modes are listed in Table 2 . In this table, only state variables of rotor angle and speed deviation are shown because these two state variables are concerned with the rotor angle stability criteria.

From the participation factor analysis, it can be determined that PSSs are installed on the dominant generators which are expressed in third column of Table 2. The type of PSS that is applied on the dominant generators will be discussed in next section.

\subsection{Application of Power System Stabilizer}

The function of PSS is to provide an additional torque to the exciter to damp out low frequency oscillations [10]. The most commonly used PSS is speed based power system stabilizer. Figure 8 illustrates the functional block diagram of PSS.

The above diagram represents a two staged PSS, consisting of a gain block, a washout circuit, dynamic compensator and limiter. In the gain block, $\mathrm{K}_{\mathrm{PSS}}$ is gain 
Table 2. Dominant generators and states for different modes in MEPE test system.

\begin{tabular}{|c|c|c|c|}
\hline Mode & Type & Dominant Generator & $\begin{array}{c}\text { Dominant States } \\
\text { (Participation Rate \%) }\end{array}$ \\
\hline 1 & Local Mode & G35 & $\begin{array}{c}\text { Rotor angle }(28.62 \%) \\
\text { Speed Deviation }(28.58 \%)\end{array}$ \\
\hline 2 & Local Mode & G5 & $\begin{array}{c}\text { Rotor angle (34.02\%) } \\
\text { Speed Deviation }(34.26 \%)\end{array}$ \\
\hline 12 & Local Mode & G4 & $\begin{array}{c}\text { Rotor angle }(32.42 \%) \\
\text { Speed Deviation }(32.76 \%)\end{array}$ \\
\hline 16 & Local Mode & G6 & $\begin{array}{c}\text { Rotor angle }(34.85 \%) \\
\text { Speed Deviation }(35.47 \%)\end{array}$ \\
\hline 19 & Local Mode & G9 & $\begin{array}{c}\text { Rotor angle }(38.82 \%) \\
\text { Speed Deviation }(39.58 \%)\end{array}$ \\
\hline 20 & Interarea Mode & G37 & $\begin{array}{c}\text { Rotor angle }(2.78 \%) \\
\text { Speed Deviation }(3.11 \%)\end{array}$ \\
\hline 30 & Interarea Mode & G32 & $\begin{array}{c}\text { Rotor angle }(11.47 \%) \\
\text { Speed Deviation }(13.02 \%)\end{array}$ \\
\hline 31 & Interarea Mode & G11 & $\begin{array}{c}\text { Rotor angle }(21.03 \%) \\
\text { Speed Deviation }(21.77 \%)\end{array}$ \\
\hline
\end{tabular}

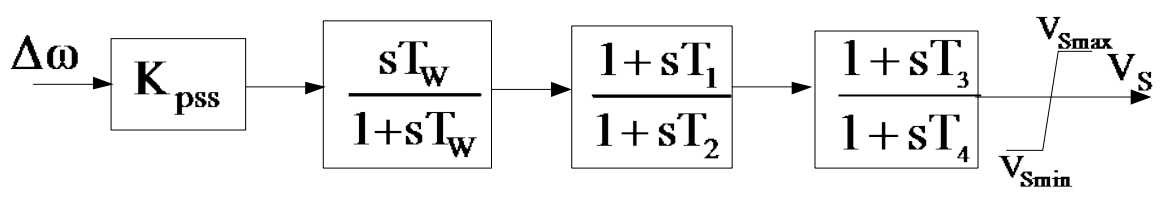

Figure 8. Block diagram of power system stabilizer.

of the PSS usually ranging from 0.01 to 50 [12]. Gain of PSS is an important factor as it is responsible for providing adequate damping torque. Damping provided by PSS is proportional to the gain until it reaches critical values, after which damping starts decreasing. Washout circuit acts as a high-pass filter. It passes all the required frequencies and eliminates steady state signals in the output of PSS which modifies generator terminal voltage. The time constant of washout filter $T_{w}$ is considered as 10 second [13]. Phase lead-lag compensation block can compensate for the lag between PSS output and electrical torque and also eliminate the delay between excitation and electrical torque. The limiter allows sufficient control range while providing satisfactory transient response. The PSS with optimal parameters are applied in the generator excitation control and their impacts on stability are assessed by eigenvalues.

\section{Simulation Results and Discussion}

\subsection{Eigenvalue Analysis of MEPE Test System with PSS}

The purpose of this section is to analyze system performances with the help of eigenvalue analysis. Obtained eigenvalues are used in assessing small signal stability of the system. When PSSs are installed on dominant generators, eigenvalue analysis is applied again through dynamic analysis channel of PSAT. Resultant 
eigenvalues of MEPE test system with PSSs (totally 554 dynamic states because of PSSs' state variables) are displayed in Figure 9.

It can be seen from Figure 9 that PSS is able to shift all real parts of electromechanical modes towards the negative left side. The system is said to be stable and the system damping ratios are enhanced by installing PSSs. The eigenvalues, the oscillation modes, their corresponding frequencies less than $2 \mathrm{~Hz}$ and damping ratios for the test system with PSSs are organized in Table 3. The oscillation modes are listed depending on the range of frequency. The significant oscillation modes, there are 8 modes out of 23 modes are tabulated in Table 3.

Comparing with the damping ratio in Table 1, it can be clearly seen that the damping ratios are greater than 0.03 with the implement of PSSs installation. It is indicated the better stabilization effects of PSS on low frequency oscillation mode.

\subsection{Dynamic Response of Generator Rotor Speed}

The power system transient stability is determined using Time Domain Simulation (TDS) routine. The system is said to be transiently stable if the TDS routes converge to the exponentially stable equilibrium point of the post-fault system [8]. In this section, response of generator rotor speed is firstly accessed to evaluate the performance of PSS.

In this study, the disturbance considered is a three phase short-circuit near Bus 18 (Thapyaywa) because three-phase fault represents the most severed isturbance for transient stability problems. The generator speed is increased because of the three phase fault and it will intend to oscillatory stability problem. Therefore, the generator speed responses of the two conditions, without and

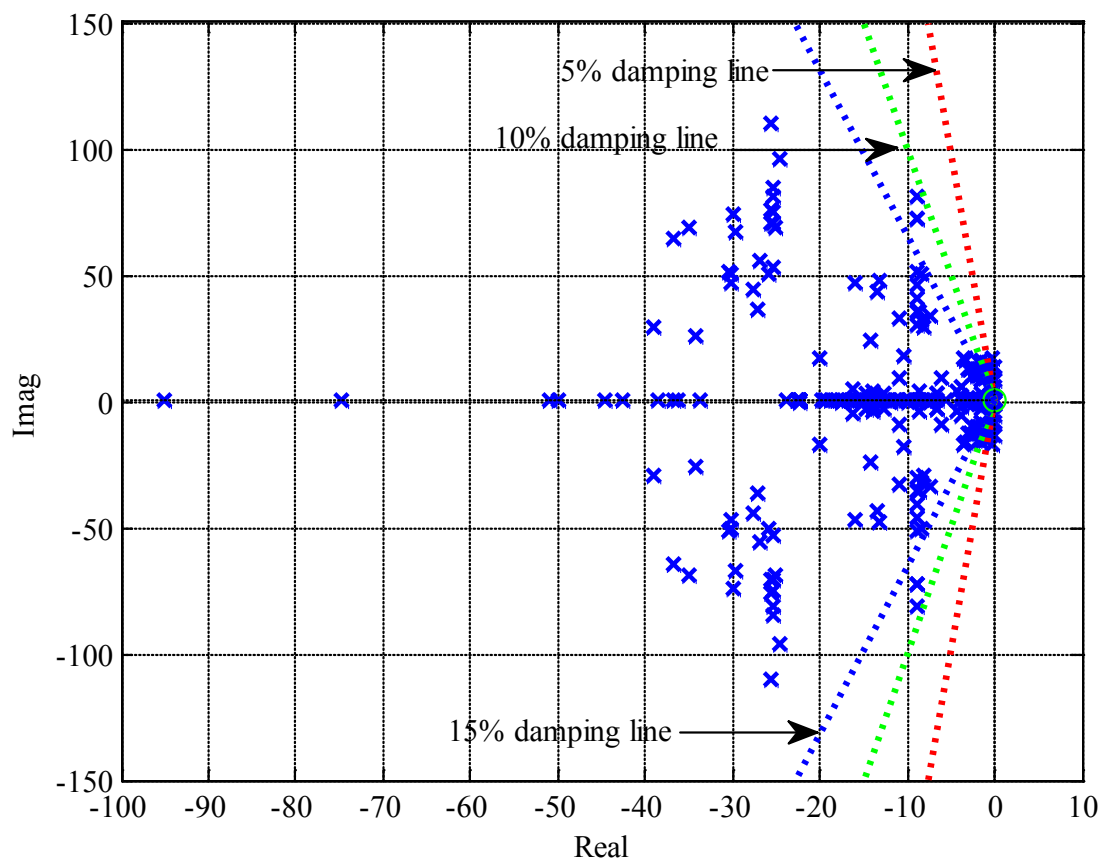

Figure 9. Eigenvalues of MEPE test system with PSS. 
Table 3. Dominant generators and states for different modes in MEPE test system.

\begin{tabular}{cccc}
\hline Mode & Eigenvalue & Frequency & Damping Ratio \\
\hline 1 & $-2.8678 \pm \mathrm{j} 12.5399$ & 1.9958 & 0.2229 \\
2 & $-2.8408 \pm \mathrm{j} 12.4357$ & 1.9792 & 0.2227 \\
5 & $-2.0541 \pm \mathrm{j} 11.2477$ & 1.7901 & 0.1797 \\
8 & $-1.8596 \pm \mathrm{j} 10.0745$ & 1.6034 & 0.1815 \\
12 & $-1.0328 \pm \mathrm{j} 9.2090$ & 1.4657 & 0.1115 \\
13 & $-0.5422 \pm \mathrm{j} 8.9655$ & 1.4269 & 0.0604 \\
16 & $-0.2882 \pm \mathrm{j} 8.5879$ & 1.3668 & 0.0335 \\
23 & $-1.4994 \pm \mathrm{j} 2.9944$ & 0.4766 & 0.4477 \\
\hline
\end{tabular}

with PSS, are preferred to detect and their simulation results are demonstrated in Figure 10 and Figure 11, respectively.

From Figure 10, it can be observed that the power system oscillations rose when applying the fault. It has been proved the oscillation of G11 is worst condition compared with other generator and otherwise the test system lost its stability.

According to results in Figure 11, it is very clear that generators with PSS improve the oscillation damping and oscillations are damp out within 3 seconds. It is evident that the generating stations with PSS provide better performance than previous case for angular stability after fault clearance due to its ability to enhance damping.

\subsection{Rotor Angle Deviation of Dominant Generators}

The variation of rotor speed can impact the rotor angle deviation because generator speed is directly related to the rotor angle. Therefore, it is required to analyze the response of rotor angle in this study. The propose PSS location and parameters has been verified by the simulation results of load angle deviation on G11 and G37 that are the most influence generator on the system stability. Figure 12 and Figure 13 show the rotor angle responses of G11 and 37 for a three phase fault in the absence and presence of PSS.

In Figure 12, the rotor angle deviation is obtained by placing PSS are also shown with bold lines. When three phase fault occur at bus 18, the rotor angle response is significantly increased with the rate of change of time. Even after clearing the fault, the rotor angle of generator fluctuates and the system operating at unstable condition. By comparing the results in Figure 12, rotor angle oscillations are eliminated when placing PSS. Therefore, PSS improves the system performance by damping out the local oscillation start from 7 second. Figure 13 depicts the variation of rotor angle of the system without and with PSS places on G37.

A three phase fault occur at $t=1$ second and cleared at 1.09 second. Before installing the PSS, the rotor angle deviation of G37 is suddenly increased during fault condition and the system is not being able to stable condition after clearing 


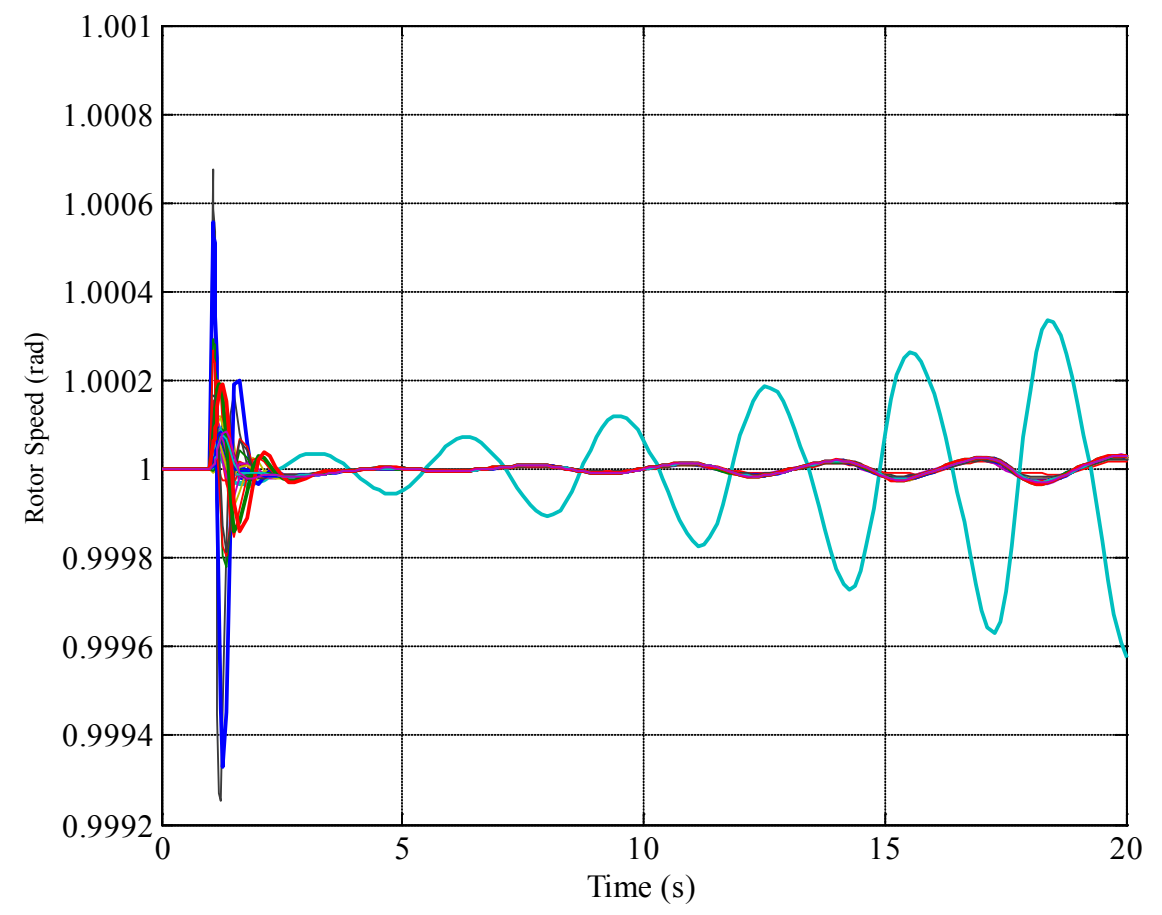

Figure 10. Response of generator rotor speed of MEPE test system without PSS.

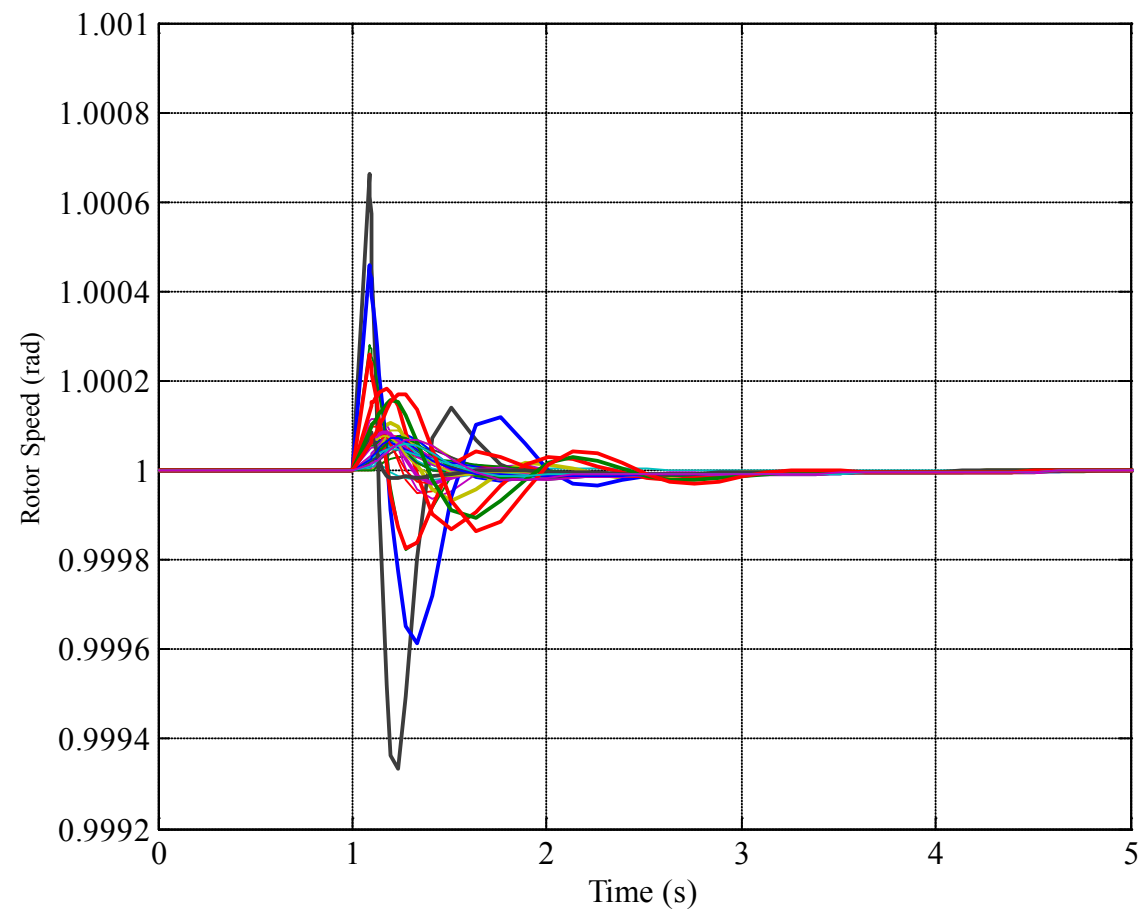

Figure 11. Speed response of generators with PSS of MEPE test system with PSS.

fault. The unstable condition is denoted by line with blue color which is represented as generator without PSS. It can be observed that the system is going unstable. When the PSS equipped on this dominant generator, the system oscillations at 4 second per cycle are decayed. It is obviously seen in Figure 13 that 


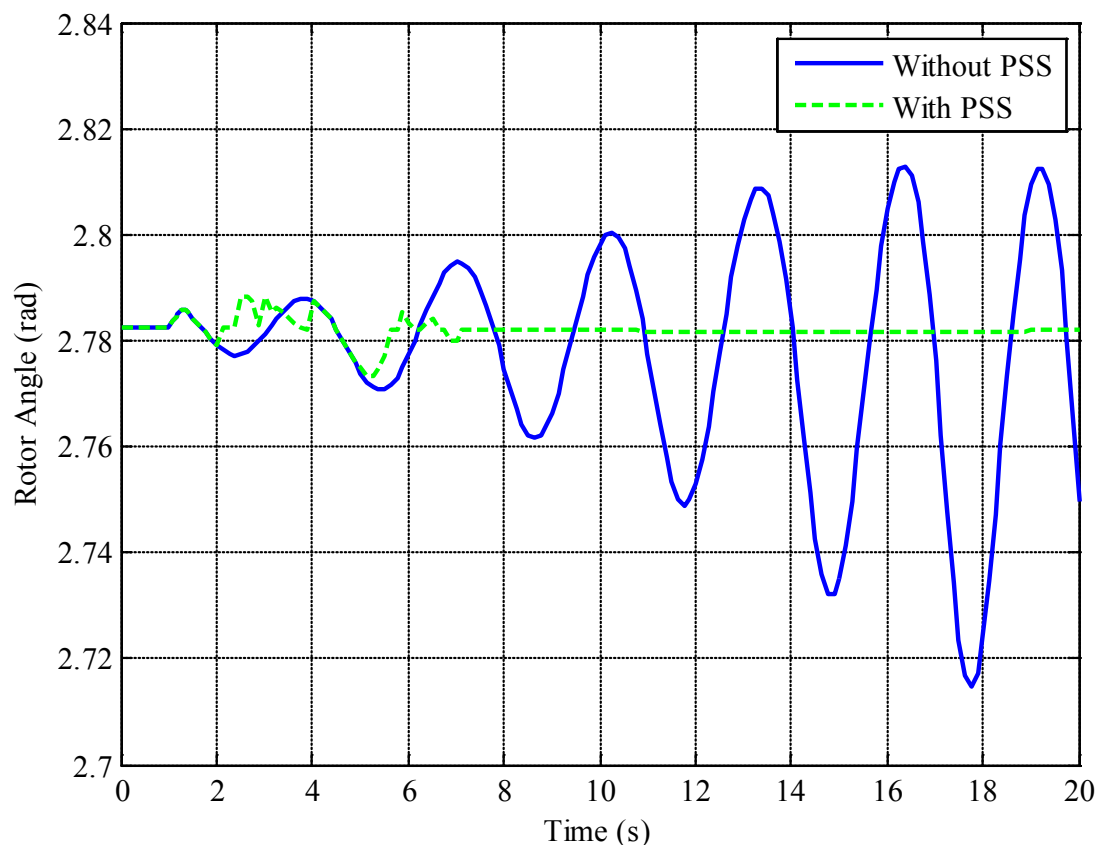

Figure 12. Rotor angle deviation of G11 without and with PSS.

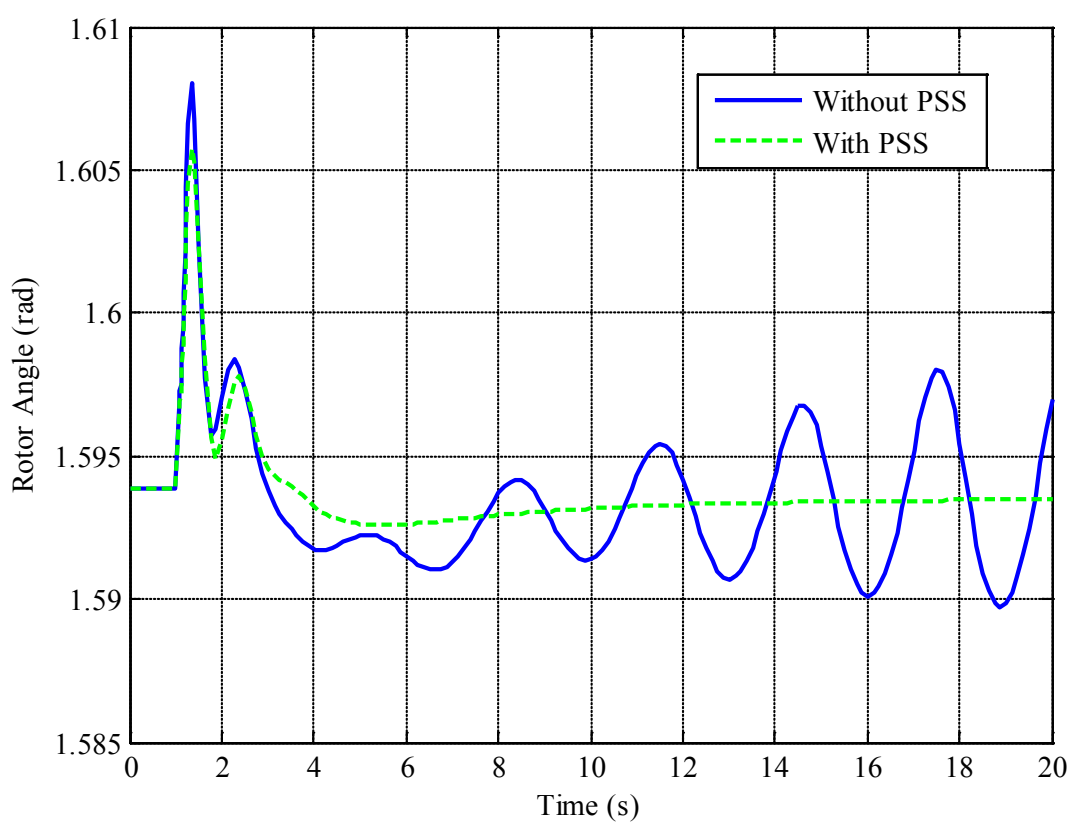

Figure 13. Rotor angle deviation of G37 without and PSS.

PSS with optimal allocation has shown better performance when compared with the response obtained by not placing PSS.

\subsection{Response of Mechanical Power Input}

In this study, the variations of mechanical power after installing PSS on dominant generator are needed to investigate. As mentioned in Section 6.1, the eight generators are candidate locations for installing PSS in MEPE test system. 
Among these, the poor situation in oscillatory stability is happened at G11 and G37. When three phase fault occur in the system, mechanical power input becomes greater and it is needed to maintain the equilibrium. Dynamic responses of mechanical power input of G11 and G37 are used to validate the performance of PSS with dominant generator. Mechanical power response of G11 is demonstrated in Figure 14 and that of G37 is illustrated in Figure 15.

By comparing the responses in Figure 14 and Figure 15, low frequency oscillations are growing after severer disturbance such as three phase fault and system without PSS indicates the state of unstable. After allocation the PSS, the amplitude of oscillations is reduced and decay nearly about 7 second. Therefore, the system becomes more stable in less time if compared to the test system without installing PSS.

\section{Conclusion}

This paper has mainly focused on the allocation of power system stabilizer (PSS) with regard to the local and inter-area modes in order to enhance the low frequency oscillation damping. The eigenvalue analysis has been utilized to analyze the damping oscillation, in which low-frequency dominant mode have been focused and to be stabilized. Participation factors have been computed effectively to place the PSS at optimal location in MEPE test system. The proposed approach has succeeded in determining the dominant generators by evaluating the participation rates in relative low frequency dominant modes. The performance of PSSs with optimal location has validated on MEPE test system. Time domain simulations and modal analysis are performed and show that the proposed

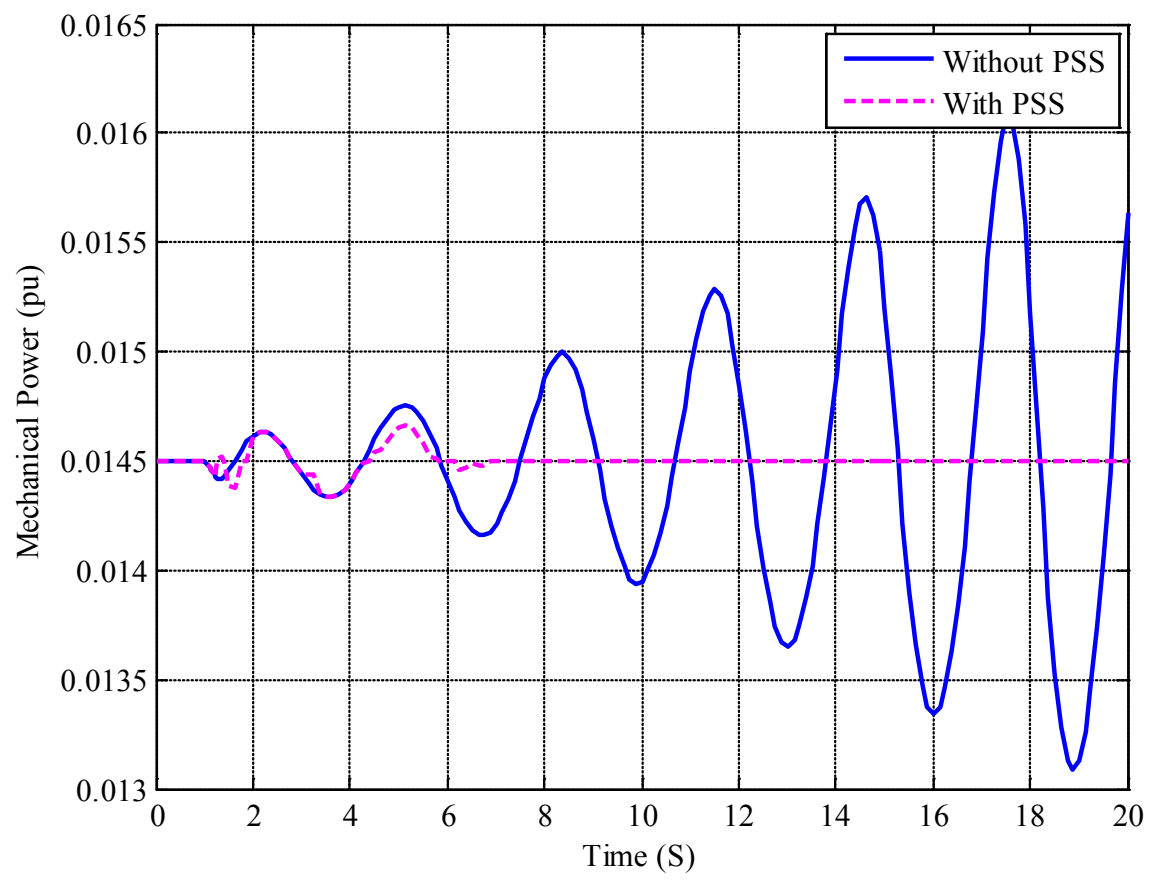

Figure 14. Mechanical power response of G11. 


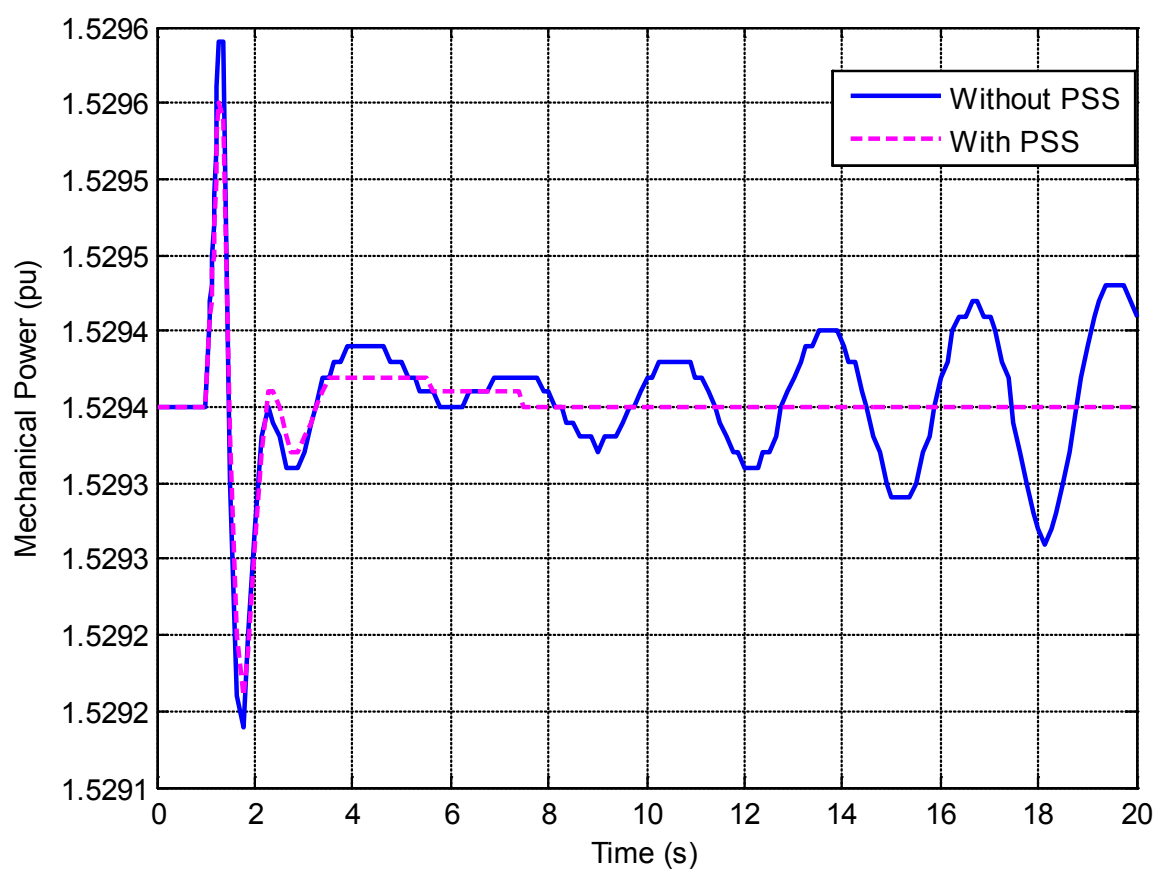

Figure 15. Mechanical power response of G37.

approach is able to find a location of PSSs with suitable parameters. The obtained simulation results show the superiority of the PSSs for improving the damping oscillations.

\section{Conflicts of Interest}

The authors declare no conflicts of interest regarding the publication of this paper.

\section{References}

[1] Dysko, A., Leithead, W.E. and O’Reilly, J. (2010) Enhanced Power System Stability by Coordinated PSS Design. IEEE Transactions on Power System, 25, 413-422. https://doi.org/10.1109/TPWRS.2009.2036704

[2] Kundur, P. (2004) Power System Stability and Control. McGraw-Hill, New York.

[3] Gibbard, M.J., Pourbeik, P. and Vowles, D.J. (2015) Small-Signal Stability, Control and Dynamic Performance of Power Systems. University of Adelaide Press, Adelaide. https://doi.org/10.20851/small-signal

[4] Prasertwong, K., Mithulananthan, N. and Thakur, D. (2010) Understanding Low-Frequency Oscillation in Power Systems. Journal of Electrical Engineering Education. https://doi.org/10.7227/IJEEE.47.3.2

[5] Rogers, G. (2000) Power System Oscillations. Kluwer, Norwell, MA. https://doi.org/10.1007/978-1-4615-4561-3

[6] Fereidouni, A.R., et al. (2013) Improvement of Low Frequency Oscillation Damping by Allocation and Design of Power System Stabilizers in the Multi-Machine Power System. Electrical Power and Energy Systems, 52, 207-220. https://doi.org/10.1016/j.ijepes.2013.03.030

[7] Milano, F. (2005) An Open Source Power System Analysis Toolbox. IEEE Transac- 
tions on Power Systems, 20, 1199-1206.

https://doi.org/10.1109/TPWRS.2005.851911

[8] Power System Analysis Toolbox, Version 2.1.9.

http://www.faradayl.ucd.ie/software.html

[9] Lin, K.M., et al. (2015) Open Source Software Based Modeling of MEPE Test System for Stability Studies. International Journal of Power and Engineering, 4, 23-31. https://doi.org/10.11648/j.ijepe.20150401.14

[10] Chomboobutrgool, Y., et al. (2012) Development and Implementation of Nordic Grid Model for Power System Small-Signal and Transient Stability Studies in Free and Open Source Software. IEEE Power and Energy Society General Meeting, San Diego, 22-26 July 2012. https://doi.org/10.1109/PESGM.2012.6344571

[11] KEPC (2011) Feasibility Study and Basic Designs for the $500 \mathrm{kV}$ Transmission System in Myanmar. Final Report, Ministry of Electric Power, Nay Pyi Taw.

[12] Dey, P., et al. (2018) Tuning of Power System Stabilizer for Small Signal Stability Improvement of Interconnected Power System. Applied Computing and Informatics, In Press. https://doi.org/10.1016/j.aci.2017.12.004

[13] Sauer, Peter, W., Pai, M.A. and Chow, J.H. (2017) Power System Dynamic and Stability. 2nd Edition, John Wiley \& Sons Ltd., UK. 